\title{
General Anesthesia
}

\section{Best evidence in anesthetic practice}

\section{Prevention: Fondaparinux is better than enoxaparin for prevention of major venous thromboembolism after orthopedic surgery}

\begin{abstract}
Article appraised
Turpie AG, Bauer KA, Eriksson BI, Lassen MR, for the Steering Committees of the Pentasaccharide Orthopedic Prophylaxis Studies. Fondaparinux vs enoxaparin for the prevention of venous thromboembolism in major orthopedic surgery: a meta-analysis of 4 randomized double-blind studies. Arch Intern Med 2002; 162: 1833-40.
\end{abstract}

\section{Structured abstract}

Question: Compared to perioperative enoxaparin, does postoperative fondaparinux decrease the incidence of postoperative venous thromboembolism (VTE)?

Design: Meta-analysis of four phase III multicentre, randomized, parallel-group, double-blind clinical trials designed a priori by one group of investigators to permit meta-analysis.

Setting: Three hundred and seventy-five centres in Argentina, Australia and New Zealand, Europe, North America, and South Africa.

Patients: Seven thousand, three hundred and fortyfour patients $\geq 18 \mathrm{yr}$ of age (mean age $68 \mathrm{yr}, 60 \%$ women) undergoing elective total hip arthroplasty (two studies), elective knee arthroplasty (one study), or hip fracture surgery (one study). Exclusion criteria were contraindications to anticoagulation therapy (active or increased risk for bleeding, planned use of indwelling intrathecal or epidural catheter, more than two attempts at neuraxial anesthesia, or hypersensitivity), addiction disorders, renal dysfunction, or required anticoagulant therapy for chronic comorbid disease. Seven thousand, two hundred and thirtyseven patients received at least one dose of the study drug and 5,385 patients completed the study. One thousand, nine hundred and fifty-nine patients (986 in the fondaparinux group; 973 in the enoxaparin group) did not receive the study drug, did not undergo the scheduled surgery, or did not undergo adequate venography for diagnosis of deep vein thrombosis (DVT).

Intervention: Three thousand, six hundred and sixty-eight patients were allocated to receive $s c$ fondaparinux $2.5 \mathrm{mg}$ once daily, beginning six hours after surgery. Three thousand, six hundred and seventy-six patients were allocated to either $s c$ enoxaparin $30 \mathrm{mg}$ twice daily, beginning 12 to $24 \mathrm{hr}$ after surgery

TABLE Eleven-day incidences of postoperative venous thromboembolism* in randomized controlled trials comparing fondaparinux and enoxaparin in major orthopedic surgery

\begin{tabular}{lllll}
\hline Type of surgery & $\begin{array}{l}\text { Fondaparinux } \\
\text { Group }\end{array}$ & $\begin{array}{l}\text { Enoxaparin } \\
\text { Group }\end{array}$ & $\begin{array}{l}\text { Relative Risk } \\
\text { (95\% confidence intervals) }\end{array}$ \\
\hline Hip arthroplasty & $85 / 1695$ & $151 / 1716$ & $0.57(0.44-0.74)$ & P-palue \\
Hip fracture repair & $52 / 626$ & $119 / 624$ & $0.44(0.32-0.59)$ & $<0.001$ \\
Knee arthroplasty & $45 / 361$ & $101 / 363$ & $0.45(0.33-0.62)$ & $<0.001$ \\
All trials & $182 / 2682$ & $371 / 2703$ & $0.49(0.42-0.58)$ & $<0.001$ \\
\hline
\end{tabular}

\footnotetext{
* Venous thromboembolic events were deep vein thrombosis or pulmonary embolus. $\dagger$ A relative risk less than zero favours the fondaparinux group; a relative risk greater than zero favours the enoxaparin group. Relative risks were calculated based on the data from the original studies.
} 
(North American regimen, two studies) or sc enoxaparin $40 \mathrm{mg}$ once daily, beginning $12 \mathrm{hr}$ before surgery with a second injection 12 to $24 \mathrm{hr}$ after surgery (European regimen, two studies).

Main outcomes: The primary efficacy outcome was the combined endpoint of 11-day DVT or pulmonary embolus. The primary safety outcome was major bleeding. Death, minor bleeding, transfusion requirements, thrombocytopenia, and other adverse events were evaluated also. Efficacy outcomes were adjudicated by a blinded committee.

Main results: Analysis was per protocol. Patient and surgical characteristics were similar between groups. The incidence of VTE was lower in the fondaparinux group $(182 / 2682)$ compared to the enoxaparin group $(371 / 2703 ; P<0.001$; Table). Major bleeding events were more frequent in the fondaparinux group $(96 / 3616)$ than in the enoxaparin group $(63 / 3621$; $P=0.008)$. The incidences of death, minor bleeding, and other adverse events did not differ between the two groups.

Conclusion: Compared to enoxaparin, fondaparinux reduced the incidence of VTE but increased the incidence of major bleeding events.

Funding: Sanofi-Synthelabo (Paris, France) and NV Organon (Oss, the Netherlands).

Correspondence: Dr. Alexander G.G. Turpie, Hamilton Health Sciences - General Hospital, Department of Medicine, 237 Barton Street East, Hamilton, Ontario, Canada L8L 2X2. E-mail: turpiea@mcmaster.ca

\section{Commentary by J.-F. Hardy}

This meta-analysis of four randomized double-blind studies compared fondaparinux, a novel synthetic anticoagulant that inhibits factor Xa via antithrombin, to two $s c$ dosages of enoxaparin for the prevention of venous thromboembolism (VTE) in major orthopedic surgery. ${ }^{1}$ The investigators must be commended for designing a priori their studies to permit meta-analysis.

Although one of the four studies did not demonstrate a benefit with fondaparinux,$^{2}$ fondaparinux reduced the pooled incidence of VTE by approximately $50 \%$. The incidence of major bleeding events and transfusions increased with fondaparinux.

How should anesthesiologists interpret these findings? How will fondaparinux influence the delivery of anesthesia in patients undergoing major orthopedic surgery?

The need for a new thromboprophylactic drug in the perioperative period remains controversial. While the incidence of venographically demonstrated VTE remained relatively high in the enoxaparin group
$(13.7 \%)$ and was reduced in the fondaparinux group $(6.8 \%)$, the incidences of symptomatic VTE were equally low in both groups $(0.4 \%$ vs $0.6 \%$ respectively). Furthermore, the incidence of fatal pulmonary embolism was the same in both groups $(0.1 \%)$. One should remember that venography remains the gold standard for the detection of deep vein thrombosis (DVT). Like most previous trials, the four studies were designed to demonstrate reductions in any VTE instead of reductions in clinically significant thromboembolic events. Nevertheless, the incidence of proximal DVT, which is more prone to embolize, was reduced significantly by fondaparinux (from $2.9 \%$ to $1.3 \%)$. This suggests that the drug may have a beneficial effect on pulmonary embolism.

Fondaparinux is a potent anticoagulant with a long half-life (approximately $15 \mathrm{hr}$ ). Prior to the phase III trials, the investigators conducted a dose ranging study and showed that a dose of $3 \mathrm{mg}$ maximized efficacy while minimizing bleeding complications in adults. ${ }^{3}$ Despite the use of $2.5 \mathrm{mg}$ in the four trials, the incidences of major bleeding events and transfusion were higher with fondaparinux (2.7\% and $53.9 \%$ respectively) than with enoxaparin ( $1.7 \%$ and $51.5 \%$ respectively). A significant relationship existed between the incidence of major bleeding and the timing (between three and nine hours after surgery) of the first fondaparinux injection, whereas efficacy was not affected by timing. These results suggest the risk of bleeding with fondaparinux may be similar to the risk with enoxaparin if the first injection of fondaparinux is given more than six hours after skin closure.

Starting fondaparinux six hours after surgery permits the use of regional anesthesia. Regional anesthesia did not influence the effectiveness of fondaparinux and was used safely in 2,496 patients. However, the protocol excluded any planned indwelling intrathecal or epidural catheter during the study treatment period and any patients with more than two attempts at neuraxial blockade. These exclusion criteria were applied because one patient developed an epidural hematoma after receiving $6 \mathrm{mg}$ of fondaparinux and five unsuccessful attempts to place an epidural catheter preoperatively during the dose finding study. ${ }^{4}$ Because of the potency and long half-life of fondaparinux, clinicians should ensure that the same conditions (no indwelling catheter, no more than two attempts at neuraxial anesthesia, initiation of treatment at least six hours after skin closure) apply when using the drug in clinical conditions.

In summary, fondaparinux reduced the incidence of venographically demonstrated VTE. While the benefit on clinically significant thromboembolic events may not be immediately apparent, fondaparinux reduced 
the incidence of proximal DVT, which is more prone to embolize. Finally, fondaparinux should be used according to the conditions of the studies included in this meta-analysis to minimize bleeding complications.

Jean-François Hardy MD FRCPC

Montréal, Québec

\section{References}

1 Turpie AG, Bauer KA, Eriksson BI, Lassen MR, for the Steering Committees of the Pentasaccharide Orthopedic Prophylaxis Studies. Fondaparinux vs enoxaparin for the prevention of venous thromboembolism in major orthopedic surgery: a meta-analysis of 4 randomized double-blind studies. Arch Intern Med 2002; 162: 1833-40.

2 Turpie AG, Bauer KA, Eriksson BI, Lassen MR; PENTATHALON 2000 Study Steering Committee.

Postoperative fondaparinux versus postoperative enoxaparin for prevention of venous thromboembolism after elective hip-replacement surgery: a randomised doubleblind trial. Lancet 2002; 359: 1721-6.

3 Turpie AG, Gallus AS, Hoek JA; Pentasaccharide Investigators. A synthetic pentasaccharide for the prevention of deep-vein thrombosis after total hip replacement. N Engl J Med 2001; 344: 619-25.

4 Hull R, Pineo G. A synthetic pentasaccharide for the prevention of deep-vein thrombosis (Letter). N Engl J Med 2001; 345: 291-2.

\section{Commentary by H.N. Lee}

What prophylaxis method should we use to prevent venous thromboembolism (VTE) in patients undergoing orthopedic surgery? Relevant issues such as efficacy, safety, cost, timing, and ability to use regional anesthetic techniques factor in this decision. Based on many randomized controlled trials (RCTs) and a costeffectiveness decision analysis, ${ }^{1}$ low molecular weight heparins have emerged as the favourite agents. However, problems with low molecular weight heparins include the need for preoperative administration in some settings, their contraindication in regional anesthesia, their increased costs compared to warfarin, especially when combined with the need for extended outpatient treatment in high-risk patients. ${ }^{2}$

Fondaparinux is a novel synthetic pentasaccharide with pure anti-factor Xa inhibitor activity that is given via daily injection postoperatively. Fondaparinux has been shown effective in four well-designed multicentre double-blind RCTs. This meta-analysis by Turpie and colleagues ${ }^{3}$ is unique because it is a pre-planned systematic review of these studies.
Thus, the usual criticisms of meta-analysis are not as relevant as the questions pertaining to VTE prevention research: are asymptomatic venogram-proven deep venous thrombi (DVT) clinically relevant? Is the 11-day study duration long enough? This meta-analysis shows that fondaparinux is clearly superior to enoxaparin in preventing these outcomes (numberneeded-to-treat [NNT] 14) in a variety of clinical settings, but at a cost of increased major bleeding (number-needed-to-harm 100). Although the authors state that there was no difference in clinically relevant bleeding, there was a significant difference in patients treated for VTE (9.7 vs 5.5\%, NNT 28) in this group of double-blind studies. An intriguing post-hoc analysis suggests that delaying the timing of the first fondaparinux injection for three to nine hours after surgery might decrease bleeding without affecting efficacy.

So is fondaparinux ready to supersede low molecular weight heparins as the prophylactic agent of choice in orthopedic surgery? Not yet. Several outstanding concerns that must be addressed include the timing of the first postoperative dose, fondaparinux's safety with the concomitant use of postoperative neuraxial or regional analgesia, the duration of treatment needed, its utility in older patients with renal dysfunction, and ultimately, its cost-effectiveness for clinically relevant outcomes. An immediate niche for fondaparinux will be its efficacy in orthopedic patients undergoing neuraxial or regional anesthesia without the need for postoperative indwelling catheters.

Hui N. Lee MD MSc FRCPC

Sault Ste. Marie, Ontario

\section{References}

1 Menzin J, Colditz GA, Regan MM, Richner RE, Oster $G$. Cost-effectiveness of enoxaparin vs low-dose warfarin in the prevention of deep-vein thrombosis after total hip replacement surgery. Arch Intern Med 1995; 155: 757-64.

2 Hull RD, Pineo GF, Stein PD, et al. Extended out-ofhospital low-molecular-weight heparin prophylaxis against deep venous thrombosis in patients after elective hip arthroplasty: a systematic review. Ann Intern Med 2001; 135: 858-69.

3 Turpie AG, Bauer KA, Eriksson BI, Lassen MR, for the Steering Committees of the Pentasaccharide Orthopedic Prophylaxis Studies. Fondaparinux vs enoxaparin for the prevention of venous thromboembolism in major orthopedic surgery: a meta-analysis of 4 randomized double-blind studies. Arch Intern Med 2002; 162: $1833-40$. 